\title{
LAGRANGIAN CONDITIONS FOR A NONSMOOTH VECTOR-VALUED MINIMAX
}

\author{
B. D. CRAVEN and D. V. LUU \\ (Received 9 September 1996; revised 8 June 1998) \\ Communicated by M. Sniedovich
}

\begin{abstract}
Lagrangian necessary and sufficient conditions for a nonsmooth vector-valued minimax in terms of Clarke's generalized Jacobians are established under suitable invexity hypotheses.

1991 Mathematics subject classification (Amer: Math. Soc.): primary 90C29; secondary 49K35.

Keywords and phrases: Nonsmooth vector-valued minimax, smooth approximation, locally invex function, pseudo-Lipschitzian multifunction.
\end{abstract}

\section{Introduction}

Minimax points, or (more restrictively) saddlepoints, occur in several optimization contexts, including stationary points that are neither maxima nor minima, saddlepoints of Lagrangians for convex minimization problems, and saddlepoints for complementary variational problems (see [1]). These ideas generalize to vector functions (see for example [8]), replacing maximum and minimum by weak maximum and weak minimum. This paper considers generalizations to Lipschitz functions, not generally differentiable at all points, by constructing approximating problems with smooth functions.

Let $f, h$ and $g$ be locally Lipschitz functions from $\mathbb{R}^{n} \times \mathbb{R}^{m}, \mathbb{R}^{m}$ and $\mathbb{R}^{n}$ into $\mathbb{R}^{r}$, $\mathbb{R}^{p}$ and $\mathbb{R}^{n}$ respectively. Let $Q \subset \mathbb{R}^{r}, T \subset \mathbb{R}^{s}$ and $S \subset \mathbb{R}^{p}$ be closed convex cones, with int $Q \neq \emptyset$. This paper considers the vector-valued minimax problem :

\section{(WMM1)}

WMIN $_{r}\left\{\right.$ WMAX $_{y} f(x, y)$ subject to $\left.-h(y) \in S\right\}$ subject to $-g(x) \in T$,

(c) 1998 Australian Mathematical Society 0263-6115/98 \$A2.00+0.00 
where WMIN denotes weak local minimum, and WMAX denotes weak local maximum, with respect to the order cone $Q$. Here a weak minimum of a vector function $\Theta$ at a point $a$ means that, for some function $\rho(\cdot)=o(\|\cdot-a\|)$,

$$
\Theta(z)-\Theta(a)-\rho(z) \notin-\text { int } Q \text { as } z \rightarrow a \text { through feasible points. }
$$

A weak maximum of $\Theta(\cdot)$ is a weak minimum of $-\Theta(\cdot)$.

Necessary and sufficient conditions for a smooth vector-valued mimimax were recently studied in [11], where under suitable invexity assumptions a weak maximum of the inner problem becomes a strong maximum with respect to a suitable basic cone $K \supset Q$. This property is not available for a nonsmooth problem. Instead, using a method of smooth approximations, based on a known technique for generalized functions (see [6], [10]), the nonsmooth problem is approximated by a smooth minimax problem, to which results for smooth vector-valued minimax (see [9]) apply.

If $S \subset \mathbb{R}^{p}$ and $K \subset \mathbb{R}^{r}$, then $\mathbb{L}(S, K)$ denotes the set of all linear mappings from $\mathbb{R}^{p}$ into $\mathbb{R}^{r}$ which map $S$ into $K$; if $b \in \mathbb{R}^{r}$, then $K_{b}:=\{\gamma(t-b): t \in K, \gamma \geq 0\}$. A convex cone $K \subset \mathbb{R}^{r}$ is called a basic cone if $K$ has exactly $r$ generators, which form a basis for $\mathbb{R}^{r}$. Let $W:=\mathbb{R}^{r} \backslash(-\operatorname{int} Q)$ and $U:=W \cap(-W) ; Q^{*}$ is the dual cone of $Q ; f(x, y)$ is a column vector, and elements of $Q^{*}$ are row vectors.

\section{Smoothed minimax problem}

For sufficiently small positive parameters $\alpha$ and $\beta$, define (see [8])

$$
\begin{gathered}
f(x, y: \alpha, \beta):=\int_{\mathbb{R}^{n}} \int_{\mathbb{R}^{n}} f(x-s, y-t) \phi(s \mid \alpha) \psi(t \mid \beta) d s d t \\
h(y: \beta):=\int_{\mathbb{R}^{n}} h(y-t) \psi(t \mid \beta) d t ; \quad g(x: \alpha):=\int_{\mathbb{R}^{n}} g(x-s) \phi(s \mid \alpha) d s ;
\end{gathered}
$$

where $d s$ and $d t$ denote Lebesgue measures on $\mathbb{R}^{n}$ and $\mathbb{R}^{m}$ respectively; $\phi(s \mid \boldsymbol{\alpha}):=$ $\alpha^{-1} \Phi\left(\alpha^{-1}\|s\|\right), \psi(t \mid \beta):=\beta^{-1} \Psi\left(\beta^{-1}\|t\|\right)$, where $\Phi$ and $\Psi$ are any fixed non-negative $C^{\infty}$-functions with supports in $(-1,1)$, scaled so that $\int_{\mathbb{R}} \Phi(z) d z=1=\int_{\mathbb{R}} \Psi(z) d z$; if $f=\left(f_{1}, f_{2}, \ldots\right)$ then $f \phi$ is the vector $\left(f_{1} \phi, f_{2} \phi, \ldots\right)$. Note that $f(\cdot, \ldots: \alpha, \beta), h(\cdot:$ $\beta$ ) and $g(\cdot: \alpha)$ are continuously differentiable (see [6]), and tend to $f(\cdot, \cdot), h(\cdot)$ and $g(\cdot)$ as $\alpha, \beta \rightarrow 0$. If $f, h, g$ have Lipschitz constants $\kappa_{f}, \kappa_{h}, \kappa_{g}$ in a neighbourhood $N_{0}$ of a point $(\bar{x}, \bar{y})$ then, for $(x, y) \in N_{0}$,

$\|g(x: \alpha)-g(x)\| \leq \int_{\mathbb{R}^{n}}\|g(x-s)-g(x)\| \phi(s \mid \alpha) d s \leq \kappa_{g} \int_{\mathbb{B}^{n}}\|s\| \phi(s \mid \alpha) d s \leq \kappa_{g}$, 
noting that $\phi(\cdot) \geq 0$ and has compact support; and similarly

$$
\|h(y: \beta)-h(y)\| \leq \kappa_{h} \beta \text {, and }\|f(x, y: \alpha, \beta)-f(x, y)\| \leq \kappa_{f}(\alpha+\beta) .
$$

By Rademacher's theorem, the Lipschitz function $g$ is Fréchet differentiable except on a set $N_{1}$ of zero measure; then (see [6])

$$
g^{\prime}(x: \alpha)=\int_{\mathbb{R}^{n}} g^{\prime}(x-s) \phi(s \mid \alpha) d s \subset C_{g}(\alpha):=\overline{\operatorname{co}}\left\{g^{\prime}(s): s \notin N_{1},\|s-x\|<\alpha\right\},
$$

where $\overline{c o}$ denotes closed convex hull, since the integrand on $N_{1}$ does not affect the integral. For sufficiently small $\alpha, C_{g}(\alpha)$ is compact, and (from [3]):

$$
\bigcap_{\alpha>0} C_{g}(\alpha)=\partial g(x)
$$

the Clarke generalized Jacobian.

Assume that (WMM1) reaches a weak local minimax at $(x, y)=(\bar{x}, \bar{y})$. Define

$$
\begin{aligned}
& \tilde{h}(y: \beta):=h(y: \beta)-h(\bar{y}: \beta)+h(\bar{y}) \\
& \tilde{g}(x: \beta):=g(x: \beta)-g(\bar{x}: \beta)+g(\bar{x}) .
\end{aligned}
$$

Then

$$
\tilde{h}(\bar{y}: \beta)=h(\bar{y}), \quad \tilde{g}(\bar{x}: \beta)=g(\bar{x}) .
$$

The problem (WMM1) is then approximated by the smooth minimax problem:

(WMM2)

$$
\begin{aligned}
& \text { WMIN }_{x}\left\{\mathrm{WMAX}_{y} f(x, y: \alpha, \beta) \text { subject to }-\tilde{h}(y: \beta) \in S\right\} \\
& \text { subject to }-\tilde{g}(x: \alpha) \in T
\end{aligned}
$$

By construction of $\tilde{g}$ and $\tilde{h}$, the point $(\bar{x}, \bar{y})$ is also feasible for (WMM2). Denote by $(\operatorname{IP}(\alpha, \beta))$ the inner problem of (WMM2).

PROPOSITION 1. Let $f$ and $g$ be locally Lipschitz functions, satisfying the stability condition:

$$
(\forall \xi \in \partial h(\bar{y})) \quad 0 \in \operatorname{int}\left[h(\bar{y})+\xi\left(\mathbb{R}^{m}\right)+S\right],
$$

and the linear growth condition, for some $0 \neq \tau \in Q *$ and some $r>0$ :

$$
(\exists \chi>0)(\forall y,\|y-\bar{y}\| \leq r,-h(y) \in S) \quad \tau f(\bar{x}, y) \leq \tau f(\bar{x}, C \bar{y})-\chi\|y-\bar{y}\| .
$$


Then, for sufficiently small $\|x-\bar{x}\|, \alpha$ and $\beta$, the problem $(\operatorname{IP}(\alpha, \beta))$ reaches a weak local maximum at a point $y=\hat{y}(x ; \alpha, \beta)$, where, for some $0<\rho<\infty$,

$$
\|\hat{y}(x ; \alpha, \beta)-\hat{y}(\bar{x} ; 0,0)\| \leq \rho\|(x, \alpha, \beta)-(\bar{x}, 0,0)\| .
$$

PROOF. Since $h$ is a locally Lipschitz function on finite dimensional spaces, the mapping $y \mapsto \partial h(y)$ is upper semicontinuous at $\bar{y}$, by [3, Proposition 2.1.5]. From this, and the hypotheses assumed, all hypotheses of [9, Lemma 4], are satisfied for the minimization of $\tau f(x, ; \alpha, \beta)$ subject to $-\tilde{h}(\cdot ; \beta) \in S$. This problem reaches a local maximum at a point $\hat{y}(x ; \alpha, \beta)$, satisfying the stated Lipschitz condition. Then $\hat{y}(x ; \alpha, \beta)$ is a weak local maximum for $(\mathrm{IP}(\alpha, \beta))$.

Let $m(x ; \alpha, \beta):=f(x, \hat{y}(x ; \alpha, \beta): \alpha, \beta) ;$ and for $\sigma \in$ int $Q$, let

$$
\hat{m}(x ; \alpha, \beta):=m(x ; \alpha, \beta)+\sigma\|x-\bar{x}\|^{2} .
$$

If $0 \neq \tau \in Q^{*}$ then $\tau \sigma>0$. Consider now the following problems:

$$
\begin{array}{ll}
(\mathrm{OP}(\alpha, \beta)) & \text { WMIN }_{x} \hat{m}(x ; \alpha, \beta) \text { subject to }-\tilde{g}(x: \alpha) \in T \\
(\mathrm{OP}(\alpha, \beta) \tau): & \operatorname{MIN}_{x} \tau \tilde{m}(x ; \alpha, \beta) \text { subject to }-\tilde{g}(x: \alpha) \in T
\end{array}
$$

PROPOSITION 2. Let $\mathrm{g}$ be locally Lipschitz. Let $\bar{x}$ be a local minimum of the problem $(\mathrm{OP}(0,0): \tau)$, and let the hypotheses of Proposition 1 hold. Then $\hat{m}(x ; \alpha, \beta)$ reaches a weak local maximum, subject to $-\tilde{g}(x: \alpha) \in T$, at a point $x=\hat{x}(\alpha, \beta)$, where $\hat{x}(\alpha, \beta) \rightarrow \bar{x}$ as $\alpha, \beta \rightarrow 0$.

Proof. By Proposition $1,(\operatorname{IP}(\alpha, \beta))$ reaches a weak local maximum $\hat{y}(x ; \alpha, \beta)$, where $\hat{y}(\cdot ; \cdot, \cdot)$ is Lipschitz at $(\bar{x} ; 0,0)$. From $(2.2)$ and the hypotheses, all the hypotheses of $[9$, Lemma 3] are satisfied for $(\mathrm{OP}(\alpha, \beta) ; \tau)$. Hence $\tau \hat{m}(\cdot: \alpha, \beta)$ reaches a local maximum, subject to $-\tilde{g}(x: \alpha) \in T$, at a point $\hat{x}(\alpha, \beta)$, where $\hat{x}(\alpha, \beta) \rightarrow \bar{x}$ as $\alpha, \beta \rightarrow 0$. Then $\hat{x}(\alpha, \beta)$ is also a weak local minimum of $(\operatorname{OP}(\alpha, \beta))$.

\section{Smoothing locally invex functions}

A differentiable function $F: \mathbb{R}^{n} \rightarrow \mathbb{R}^{r}$ is invex at $p \in \mathbb{R}^{n}$ with respect to the order cone $Q \subset \mathbb{R}^{n}$ if, for some scale function $\eta(x, p)$,

$$
(\forall x) F(x)-F(p)-F^{\prime}(p) \eta(x, p) \in Q
$$


It can be assumed that, for $p$ fixed, $\eta(x, p)=x-p+o(\|x-p\|)$, so $\eta(\cdot, p)$ is continuous, and $\eta(p, p)=0$. A Lipschitz function $F: \mathbb{R}^{n} \rightarrow \mathbb{R}^{r}$ is $Q$-invex at $p$ if

$$
(\forall x)(\forall \zeta \in F(p)) F(x)-F(p)-\zeta \eta(x, p) \in Q,
$$

where $\partial F(p)$ is the Clarke generalized Jacobian of $F$ at $p$. By Rademacher's theorem, $F$ is differentiable except on a set $N_{0}$ of zero Lebesgue measure.

DEFINITION 1. A Lipschitz function $F: \mathbb{R} \rightarrow \mathbb{R}^{r}$ is $Q$-invex around $p$ if, for some continuous scale function $\eta(\cdot, \cdot)$, and each $u$ in some neighbourhood of $p$ with $u \notin N_{0}$,

$$
(\forall x) F(x)-F(u)-F^{\prime}(u) \eta(x-u+p, p) \in Q .
$$

Clearly, $Q$-invex around $p$ implies $Q$-invex at $p$.

Proposition 3. Let $F(\cdot)$ be $Q$-invex around $p$. Then the smoothed function

$$
F(\cdot: \alpha):=\int_{\mathbb{R}^{n}} F(x-s) \phi(s \mid \alpha) d s,
$$

with $\phi$ chosen as in Section 2, is $Q$-invex at $p$, with the same scale function as $F(\cdot)$, whenever $\alpha$ is sufficiently small.

REMARKS. This conclusion, required in Theorem 1 , would not hold if $Q$-invex around $p$ is weakened to invex at each point in a neighbourhood of $p$, because a sum of invex functions is not necessarily invex, if the scale functions are different.

Invex around $p$ holds, in particular, for a $C^{1}$-function $F$ if invexity at the point $p$ is strengthened to

$$
(\forall x) F(x)-F(p)-F^{\prime}(p) \eta(x, p)-\sigma\|x-p\|^{2} \in Q,
$$

where $\sigma \in$ int $Q$. For then

$$
(\forall x) F(x)-F(p)-F^{\prime}(p) \eta(x, p) \in \text { int } Q,
$$

so that, for sufficiently small $\delta$,

$$
(\forall x)(\forall u,\|u-p\|<\delta) \quad F(x)-F(u)-F^{\prime}(u) \eta(x-u+p, p) \in Q .
$$

For a $C^{\infty}$ function $F$, shifting origins to make $p=0$ and $F(p)=0$, consider

$$
F(z)=A z+\frac{1}{2} z^{T} B \cdot z \quad \text { and } \quad \eta(z, 0)=z+\frac{1}{2} z^{T} D \cdot z
$$

up to quadratic terms. Here $B$. represents the set of Hessian matrices, $B_{k}$ say, for the components of $F$, and similarly for $D$.. Substitution in the definition then shows that invex around 0 requires the matrices $B .-\left(A-u^{T} B\right.$. $) D$. to be positive semidefinite, for sufficiently small $\|u-\|$, where $A D$. represents the matrices $\Sigma_{s} A_{s k} D_{k}$. Hence the matrices $B .-A D$. must be positive definite, and this in turn implies that $F$ is invex around 0 . 
PROOF OF PROPOSITION 3. Using the differentiability of $F(\cdot: \alpha)$ and the Lipschitz property of $F(\cdot)$,

$$
F^{\prime}(\cdot: \alpha)=\int_{\mathbb{R}^{n}} F^{\prime}(x-s) \phi(s \mid \alpha) d s,
$$

since $F^{\prime}(x-s)$ is only undefined on a set of zero measure. Hence, for $\left\{u_{j}\right\} \rightarrow p$ with $u_{j} \notin N_{0}$, and noting that $\phi(\cdot \mid \alpha) \geq 0$,

$$
\begin{aligned}
& F\left(u_{j}+y: \alpha\right)-F\left(u_{j}: \alpha\right)-F^{\prime}\left(u_{j}: \alpha\right) \eta(p+y, p) \\
& \quad=\int_{\mathbb{R}^{n}}\left[F\left(u_{j}+y-s\right)-F\left(u_{j}-s\right)-F^{\prime}\left(u_{j}-s\right) \eta(p+y, p)\right] \phi(s \mid \alpha) d s,
\end{aligned}
$$

in which $[\ldots] \in Q$ for almost all $s$, provided that $u_{j}$ and $u_{j}-s$ are in a suitable neighbourhood of $p$. Consequently,

$$
F\left(u_{j}+y: \alpha\right)-F\left(u_{j}: \alpha\right)-F^{\prime}\left(u_{j}: \alpha\right) \eta(p+y, p) \in Q,
$$

which implies that

$$
F(p+y: \alpha)-F(p: \alpha)-F^{\prime}(p: \alpha) \eta(p+y, p) \in Q .
$$

REMARK. Suppose that $F: \mathbb{B}^{n} \rightarrow \mathbb{R}^{r}$ (where $r<n$ ) is invex at each point in a ball $B$ with centre $p$, with respect to an order cone $Q \subset \mathbb{R}^{r}$. If $F$ is differentiable at $u \in B \backslash\{p\}$ then:

$$
\begin{aligned}
F(u+z: \alpha)-F(u: \alpha)=\int[F(u+z-s)-F(u-s)] \phi(s \mid \alpha) d s \\
\geq \int F^{\prime}(u-s) \omega(z, u-s) \phi(s \mid \alpha) d s \\
\quad(\text { where } \omega(z, u-s):=\eta(z+u-s, s)) \\
=F^{\prime}(u \mid \alpha) \theta(z, u)
\end{aligned}
$$

if there exists $\theta(z, u)$ satisfying the linear equation

$$
\mathbb{M} \theta(z, u) \equiv\left[\int F^{\prime}(u-s) \phi(s \mid \alpha) d s\right] \theta(z, u)=\int F^{\prime}(u-s) \omega(z, u)-\phi(s \mid \alpha) d s .
$$

Consider the hypothesis that for each sufficiently small $\delta>0$, there exists some $s$, with $\|s\|<\delta$, such that $F^{\prime}(u-s)$ has full rank. This implies that, for some $\phi(\cdot) \geq 0$ with support in a ball of radius $\delta, M$ has full rank; hence $\theta(z, u)$ exists, and $\theta(z, p)$ as a limiting case. Thus $F(\cdot: \alpha)$ is invex, as required in the proof of Theorem 1.

If $F$ is differentiable at $p$, and $p$ is a Karush-Kuhn-Tucker point for the minimization of $F_{1}(\cdot)$ subject to $F_{j}(\cdot) \leq 0(j=2,3, \ldots, r)$, then $F^{\prime}(p)$ does not have full rank. But $F^{\prime}(p-s)$ may have full rank, for small $\|s\|>0$. 


\section{Necessary conditions for a weak minimax}

Assume that the inner problem of (WMM1):

$$
\left(\operatorname{IP}_{x}\right): \text { WMAX }_{y} f(x, y) \text { subject to }-h(y) \in S:=\mathbb{R}_{+}^{p} \text {, }
$$

reaches a (non-unique) weak local maximum at $y=\hat{y}(x)$, with $\hat{y}(\vec{x})=\bar{y}$ and constraints of $\left(\mathrm{IP}_{\bar{x}}\right)$ are active at $\bar{y}$. Following [11], construct the problem $\left(\mathrm{MP}_{\bar{x}}\right)$ by adjoining $p-k$ additional constraints:

$$
\phi^{(j)}(y-\bar{y}):=a_{j}^{T}(y-\bar{y})+\frac{1}{2}(y-\bar{y})^{T} A_{j}(y-\bar{y}) \leq 0 \quad(j=1,2, \ldots, p-k)
$$

to $\left(\mathrm{IP}_{\bar{x}}\right)$. The matrices $A_{j}$ are chosen (see [11], and (iii) in Theorem 1 below) so that the $\phi^{(j)}$ satisfy an invex property. Then $\bar{y}$ is also a weak maximum of $\left(\mathrm{MP}_{\bar{x}}\right)$. Another similar set of functions

$$
\psi^{(j)}(y-\bar{y}):=b_{j}^{T}(y-\bar{y})+\frac{1}{2}(y-\bar{y})^{T} B_{j}(y-\bar{y}) \leq 0 \quad(j=1,2, \ldots, s-l)
$$

is also required in Theorem 2 , with the $B_{j}$ chosen to satisfy an invex property. Note that $\phi^{(j)}(0)=0$ and $\psi^{(j)}(0)=0$. (These $\phi^{(j)}$ and $\psi^{(j)}$ are unrelated to the mollifier functions $\phi$ and $\psi$ in section 2.)

Under the hypotheses of Proposition 1, the smoothed problem $(\operatorname{IP}(\alpha, \beta))$ reaches a weak local maximum at $y=\hat{y}(x ; \alpha, \beta)$, where $\hat{y}(\cdot ; \cdot, \cdot)$ satisfies a Lipschitz condition. Consider then the following smoothed version of $\left(\mathrm{MP}_{x}\right)$ :

$$
\text { WMAX }_{y} f(x, y: \alpha, \beta) \text { subject to }
$$

$(\operatorname{MIP}(\alpha, \beta))$ :

$$
\begin{gathered}
-\tilde{h}^{(i)}(y: \beta) \leq 0 \quad(i=1,2, \ldots, p) \\
\phi^{(j)}(y-\hat{y}(x ; \alpha, \beta)) \leq 0 \quad(j=1,2, \ldots, p-k) .
\end{gathered}
$$

Then $\hat{y}(x ; \alpha, \beta)$ is also a weak local maximum for $(\operatorname{MIP}(\alpha, \beta))$. Under a constraint qualification, weak Karush-Kuhn-Tucker conditions [5, Theorem 1] hold:

(WKT):

$$
\begin{aligned}
& \tau(x ; \alpha, \beta) f_{y}(x, \hat{y}(x: \alpha, \beta): \alpha, \beta) \\
& \quad=\Sigma_{i} \lambda_{i}(x ; \alpha, \beta) \tilde{h}_{y}^{(i)}(\hat{y}(x ; \alpha, \beta): \beta)+\Sigma_{j} \mu_{j}(x ; \alpha, \beta) a_{j}^{T} ; \\
& \quad \lambda_{i}(x ; \alpha, \beta) \tilde{h}^{(i)}(\hat{y}(x ; \alpha, \beta): \beta)=0 ;
\end{aligned}
$$

for some nonnegative Lagrange multipliers $\tau(x ; \alpha, \beta), \lambda_{i}(x ; \alpha, \beta), \mu_{j}(x ; \alpha, \beta)$; the summation $\Sigma_{i}$ is over active constraints (thus $h^{(i)}(\tilde{y})=0$ ), and $\Sigma_{j}$ is over $j=$ $1, \ldots, k-p$. 
THEOREM 1. Let $(\bar{x}, \bar{y})$ be a local weak minimax for (WMM1). Assume that:

(i) the hypotheses of Propositions 1 and 2 hold;

(ii) for each $\xi_{i} \in \partial h^{(i)}(\bar{y})$, the set of gradients $\xi_{i}$, for those constraints for which $h^{(i)}(\bar{y})=0$, together with the gradients $a_{j}^{T}(j=1, \ldots, p-k)$ of the adjoined constraints, form a linearly independent set;

(iii) the vector function $H(\cdot)$, comprising $-f(x, \cdot)$, those $h^{(i)}(\cdot)$ for which $h^{(i)}(\bar{y})=$ 0 , and $\phi^{(j)}(\cdot)(j=1,2, \ldots, k-p)$, is $Q \times \mathbb{R}_{+}^{p}$-invex around each point $y^{\prime}$ in a neighbourhood of $\bar{y}$, whenever $\|x-\bar{x}\|$ is sufficiently small;

(iv) the Lagrange multipliers in (WKT) are continuous at $(\bar{x}, 0,0)$;

(v) the Lagrange multipliers for $(\mathrm{OP}(\alpha, \beta))$ are continuous at $(0,0)$;

(vi) $(\forall \eta \in \partial g(\bar{x})) 0 \in$ int $\left[g(C x)+\eta\left(\mathbb{R}^{n}\right)+T\right]$.

Then, for some basic cone $K \supset Q$, and some $\delta>0$,

$$
\begin{gathered}
(\forall x,-g(x) \in T,\|x-\bar{x}\|<\delta)\left(\exists \hat{\Lambda}(x) \in \mathbb{R}\left(\mathbb{R}_{+}^{p}, K\right),\right. \\
\left.\hat{M}(x) \in \mathbb{L}\left(\mathbb{R}_{+}^{p-k}, K\right), \bar{\pi} \in \mathbb{L}\left(T_{-g(\bar{x})}, Q\right), \bar{\xi} \in \partial_{x} f(\bar{x}, \bar{y}), \bar{\eta} \in \partial g(\bar{x})\right), \\
0 \in \partial_{y}[f(x, \hat{y}(x))-\hat{\Lambda}(x) h(\hat{y}(x))]-\hat{M}(x) a ; \hat{\Lambda}(x) h(\hat{y}(x))=0 ; \operatorname{ran}[\hat{\xi}+\hat{\pi} \hat{\eta}] \subset U .
\end{gathered}
$$

PROOF. From (iii) and Proposition 3, the vector function $\hat{H}(\cdot)$, comprising the objective $-f(x, \cdot: \alpha, \beta), \tilde{h}^{(i)}(\cdot ; \beta)$ for the constraints for which $h^{(i)}(\bar{y})=0$, and $\phi^{(j)}(\cdot)$ for $j=1,2, \ldots, k-p$, is $Q \times \mathbb{R}_{+}^{p}$-invex at each $y^{\prime}$ in a neighbourhood of $\bar{y}$, whenever $\|x-\bar{x}\|$ is sufficiently small. From Proposition 1 , the problem $(\operatorname{IP}(\alpha, \beta))$ reaches a weak local maximum at $y=\hat{y}(x ; \alpha, \beta)$, whenever $\alpha, \beta$ and $\|x-\bar{x}\|$ are sufficiently small. From (ii), the Robinson stability condition (see [14], and [16] for the nonsmooth generalization) holds for $\left(\mathrm{IPC}_{x}\right)$. Since this condition requires 0 in the interior of a set involving gradients, and the Clarke generalized subdifferentials are upper semicontinuous, the stability condition extends to $(\operatorname{MIP}(\alpha, \beta))$ for sufficiently small $\|x-\bar{x}\|$. Then, applying [11, Proposition 1] to $(\operatorname{MIP}(\alpha, \beta))$, which requires the invexity hypothesis (iii), there is some basic cone $K \supset Q$, such that the strong Karush-Kuhn-Tucker conditions hold:

$$
\begin{gathered}
f_{y}(x, \hat{y}(x ; \alpha, \beta): \alpha, \beta)-\Lambda(x ; \alpha, \beta) \tilde{h}_{y}(\hat{y}(x ; \alpha, \beta): \beta)-M(x ; \alpha, \beta) a=0 \\
\Lambda(x ; \alpha, \beta) \tilde{h}(\tilde{y}(x ; \alpha, \beta): \beta)=0
\end{gathered}
$$

for some Lagrange multipliers $\Lambda(x ; \alpha, \beta) \in \mathbb{L}\left(\mathbb{R}_{+}^{p}, K\right)$ and $M(x ; \alpha, \beta) \in \mathbb{L}\left(\mathbb{R}_{+}^{p-k}, K\right)$. From Proposition 2, the function $\hat{m}(\cdot ; \alpha, \beta)$ from Section 2 reaches a weak local minimum, subject to $-\tilde{g}(\cdot ; \alpha) \in T$, at $\hat{x}(\alpha, \beta)$, where $\hat{x}(\alpha, \beta) \rightarrow \bar{x}$ as $\alpha, \beta \rightarrow 0$.

From (vi), the smoothed function $\tilde{g}$ satisfies the Robinson condition:

$0 \in$ int $\left[\tilde{g}(\bar{x} ; \alpha)+\tilde{g}_{x}(\bar{x} ; \alpha)\left(\mathbb{R}^{n}\right)+T\right]$ whenever $\alpha$ is sufficiently small. 
From [6, Theorem 1], there exists a Lagrange multiplier $\pi(\alpha, \beta) \in \mathbb{L}\left(\mathbb{R}^{s}, \mathbb{R}^{r}\right)$, satisfying

$$
\operatorname{ran}\left[\hat{m}_{x}(\bar{x} ; \alpha, \beta)+\pi(\alpha, \beta) \tilde{g}_{x}(\bar{x} ; \alpha)\right] \subset U ; \pi(\alpha, \beta)\left(T_{-\bar{g}(\bar{x} ; \alpha)}\right) \subset Q
$$

From [11, Proposition 2], $m_{x}(\bar{x} ; \alpha, \beta)=f_{x}(\bar{x}, \bar{y}: \alpha, \beta)$. Combining these,

$$
\operatorname{ran}\left[f_{x}(\bar{x}, \bar{y}: \alpha, \beta)+\pi(\alpha, \beta) \tilde{g}_{x}(\bar{x} ; \alpha)\right] \subset U
$$

Letting $\alpha, \beta \rightarrow 0, \Lambda(x ; \alpha, \beta) \rightarrow \tilde{\Lambda}(x)$ and $M(x ; \alpha, \beta) \rightarrow \tilde{M}(x)$ (these limits existing by (iv)), $\pi(\alpha, \beta) \rightarrow \bar{\pi}$ (by hypothesis (v)), the left side of (4.4) tends to an element of $\partial_{y}(f-\hat{\Lambda}(x) h-\hat{M}(x) a)(x, \hat{y}(x)), f_{x}(\bar{x}, \bar{y} ; \alpha, \beta)$ and $\tilde{g}_{x}(\bar{x} ; \alpha)$ tends to elements of $\left.\partial_{x} f \bar{x}, \bar{y}\right)$ and $\partial g(\bar{x})$. So the results follow as limits from (4.4) and (4.5).

REMARKS. If hypothesis (vi) is replaced by the hypothesis that, for each $\eta_{i} \in$ $\partial g_{i}(\bar{x})$, the set of $\eta_{i}$ for the active constraints, together with $b_{j}^{T}(j=1,2, \ldots, s-l)$, is linearly independent, then the conclusion holds in which the inclusion $\operatorname{ran}[\bar{\xi}+\bar{\pi} \bar{\eta}] \subset U$ is replaced by

$$
0 \in \partial_{x}[f(\bar{x}, \bar{y})+\bar{\pi} g(\bar{x})]+\bar{\Xi} b, \bar{\pi} g(\bar{x})=0
$$

for $\left.\bar{\pi} \in \mathbb{L}\left(\mathbb{R}_{+}^{s}\right), H\right), \bar{\Xi} \in \mathbb{L}\left(\mathbb{R}^{s-l}, H\right)$, where $H$ is a basic cone satisfying $Q \subset K \subset H$.

Theorem 1 assumes that Lagrange multipliers are continuous functions of smoothing parameters. Consider strong Karush-Kuhn-Tucker conditions expressed as:

$$
f_{y}(x, \hat{y}(x ; \alpha, \beta): \alpha, \beta)=\Delta(x ; \alpha, \beta) k_{y}(\hat{y}(x ; \alpha, \beta): \alpha, \beta),
$$

where $\Delta$ comprises $\Lambda$ and $M$, and $k_{y}$ comprises $\tilde{h}_{y}$ and $a$. In case the $p \times m$ matrix $k_{y}$ has full rank, and $p \leq n$, then the linear equation for $\Delta$ is solvable, for $\Delta$ a continuous function of $\alpha, \beta$.

\section{Sufficient conditions}

Recall [13] that a multifunction $\Phi: \mathbb{R}^{n} \rightarrow \mathbb{R}^{m}$ is called pseudo-Lipschitzian around $(\bar{x}, \bar{y}) \in \operatorname{gr} \Phi$ (the graph of $\Phi$ ) with modulus $c>0$ if there exist neighbourhoods $U$ of $\bar{x}$ and $V$ of $\bar{y}$ such that

$$
\left(\forall x, x^{\prime} \in U\right) \Phi\left(x^{\prime}\right) \cap V \subset \Phi(x)+c\left\|x-x^{\prime}\right\| B_{1}(0) .
$$


Here $B_{1}(0)$ is the unit closed ball. Denote the Clarke generalized Jacobian with respect to $x$ and $y$ by $\partial_{x}$ and $\partial_{y}$ and let $\mathbb{N}(v, \Omega)$ denote the Clarke normal cone to a set $\Omega$ at a point $v \in \Omega$. Define the Lagrangian:

$L(x, y ; \Lambda, M, \Pi, \Xi):=f(x, y)-\Lambda h(y)-M \phi(y-C y)+\Pi g(x)+\Xi \psi(x-\bar{x})$.

A function $F(\cdot)$ reaches a (local) strong minimum at $\bar{x}$ with respect to a cone $H$ (see [4]) if $F(x)-F(\bar{x}) \in H$ for all feasible $x$ near $\bar{x}$.

THEOREM 2. Let $(\bar{x}, \bar{y})$ be a feasible point for (WMM1), where $k \leq p$ constraints from $-h(\cdot) \in S$ are active at $\bar{y}$, and $l \leq s$ constraints from $-g(\cdot) \in T$ are active at $\bar{x}$. Assume that:

(a) For some closed convex cones $K$ and $H, Q \subset K \subset H$, and

$$
\begin{gathered}
\left(\exists \tilde{\Lambda} \in \mathbb{L}\left(S_{-h(\bar{y})}, K\right), \tilde{M} \in \mathbb{L}\left(\mathbb{R}_{+}^{p-k}, K\right), \tilde{\Pi} \in \mathbb{L}\left(T_{-g(\bar{x})}, H\right), \tilde{\Xi} \in \mathbb{L}\left(\mathbb{R}_{+}^{s-1}, H\right)\right) \\
(0,0) \in \partial_{x} L(\bar{x}, \bar{y} ; \tilde{\Lambda}, \tilde{M}, \tilde{\Pi}, \tilde{\Xi}) \times \partial_{y} L(\bar{x}, \bar{y} ; \tilde{\Lambda}, \tilde{M}, \tilde{\Pi}, \tilde{\Xi})
\end{gathered}
$$

(here $\partial_{x} L$ is independent of $(\Lambda, M)$, and $\partial_{y} L$ is independent of $(\Pi, \Xi)$ );

(b) For sufficiently small $\|x-\bar{x}\|$, the vector function comprising $-f(x, \cdot), h(\cdot)$, and $\phi^{(j)}(\cdot-\bar{y})(j=1,2, \ldots, p-k)$ (as in (4.2)) is $Q \times S \times \mathbb{R}_{+}^{p-k}$-invex at each point $y^{\prime}$ in some neighbourhood of $\bar{y}$, with scale function independent of $x$, and the vector function comprising $f(\cdot, \bar{y}), g(\cdot)$, and $\psi^{(j)}(\cdot-\bar{x})(j=1,2, \ldots, s-l)$ is $Q \times T \times \mathbb{R}_{+}^{s-l}$-invex at $\bar{x}$;

(c) $(\zeta, 0) \in \mathbb{N}((\bar{x}, \bar{z}), \Omega) \Rightarrow \zeta=0$,

where

$$
\begin{aligned}
\Omega:= & \operatorname{ker} \partial_{y} L \cap\left(\mathbb{R}^{n} \times \operatorname{ker}[\Lambda h(y)+M \phi(y-\bar{y})]\right) \cap \\
& \left(\left[\mathbb{R}^{n} \times \mathbb{R}^{m} \times\left\{(\Lambda, M): \Lambda(S)+M\left(\mathbb{R}_{+}^{p-k}\right) \subset K\right\}\right) .\right.
\end{aligned}
$$

Then $(\bar{x}, \bar{y})$ is a local strong minimax for (WMM1), with the additional constraints $\phi^{(j)}(y-\bar{y}) \leq 0(j=1,2, \ldots, p-k)$ and $\psi^{(j)}(x-\bar{x}) \leq 0(j=1,2, \ldots, s-l)$ adjoined, with respect to the order cones $K$ and $H$.

PROOF. Define a multifunction $\Phi$ by

$$
\begin{aligned}
\Phi(x):=\left\{z=(y, \Lambda, M): 0 \in \partial_{y} L(x, y ; \Lambda, M, \Pi, \Xi), \Lambda h(y)+M \phi(y-\bar{y})\right. & =0, \\
\Lambda(S)+M\left(\mathbb{R}_{+}^{p-k}\right) & \subset K\},
\end{aligned}
$$


noting that $\partial_{y} L(\cdot)$ does not involve $\Pi$ and $\Xi$. From hypothesis $(\mathrm{a}), \bar{z}:=(\bar{y}, \tilde{\Lambda}, \tilde{M}) \in$ $\Phi(\bar{x})$. From definition of $\Phi(\cdot), \operatorname{gr} \Phi=\Omega$. Using hypothesis (c),

$$
(\zeta, 0) \in \mathbb{N}((\bar{x}, \bar{z}), \operatorname{gr} \Phi) \Rightarrow \zeta=0 .
$$

According to Aubin [1] and Rockafellar [15], this condition ensures that $\Phi$ is pseudoLipschitzian. Hence, whenever $\|x-C \bar{x}\|$ is sufficiently small, $\Phi(x) \neq \emptyset$, (as shown in the proof of $[10$, Theorem 3$]$, thus

$$
(\exists \hat{z}(x)=(\hat{y}(x), \hat{\Lambda}(x), \hat{M}(x)) \ni \Phi(x)) \hat{z}(\bar{x})=(\bar{y}, \tilde{\Lambda}, \tilde{M}) .
$$

From this, with hypothesis (b), if $-h(y) \in S, \phi^{(j)}(y-\bar{y}) \leq 0(j=1,2, \ldots, p-k)$, then

$$
\begin{aligned}
& f(x, \hat{y}(x))-f(x, y) \\
& =L(x, \hat{y}(x) ; \hat{\Lambda}(x), \hat{M}(x), \Pi, \Xi)-L(x, y ; \hat{\Lambda}(x), \hat{M}(x), \Pi, \Xi) \\
& \quad+\hat{\Lambda}(x)[-h(y)+h(\hat{y}(x))]+\hat{M}(x)[-\phi(y-\bar{y})+\phi(\hat{y}(x)-\bar{y})] \\
& \quad \in-A(x) \zeta(y, \hat{y}(x))+Q+K \subset K,
\end{aligned}
$$

where $A(x) \in \partial_{y} L(x, \hat{y}(x) ; \hat{\Lambda}(x), \hat{M}(x), \Pi, \Xi), \zeta(y, \hat{y}(x))$ is a scale function from the invex hypothesis (b), $\phi:=\left(\phi^{(1)}, \ldots, \phi^{(p-k)}\right)$, and the values of $\Pi$ and $\Xi$ do not matter in this calculation. Hence $\hat{y}(x)$ is a strong maximum, with respect to the cone $K$, for the inner problem of (WMM1) with additional constraints $\phi^{(j)}(y-\bar{y}) \leq$ $0(j=1,2, \ldots, p-k)$. From $\hat{z}(x) \in \Phi(x)$ and hypotheses (a) and (b), if $\|x-\bar{x}\|$ is sufficiently small, $-g(x) \in T$, and $\psi^{(j)}(x-\bar{x}) \leq 0(j=1, \ldots, s-l)$ then

$$
\begin{aligned}
& f(x, \hat{y}(x))-f(\bar{x}, \bar{y}) \\
& =f(x, \hat{y}(x))-f(x, \hat{y})+f(x, \bar{y})-f(\bar{x}, \bar{y}) \\
& =\quad L(x, \hat{y}(x) ; \hat{\Lambda}(x), \hat{M}(x), \Pi, \Xi)-L(x, \hat{y} ; \hat{\Lambda}(x), \hat{M}(x), \Pi, \Xi) \\
& \quad+L(x, \bar{y} ; \tilde{\Lambda}, \tilde{M}, \tilde{\Pi}, \tilde{\Xi})-L(\bar{x}, \bar{y} ; \tilde{\Lambda}, \tilde{M}, \tilde{\Pi}, \tilde{\Xi}) \\
& \quad-\hat{\Lambda}(x) h(\bar{y})-\hat{M}(x) \phi(0)+\hat{\Lambda}(x) h(\hat{y}(x))+\hat{M}(x) \phi(\hat{y}(x)-\bar{y}) \\
& \quad+\tilde{\Pi}[-g(x)+g(\bar{x})]+\tilde{\Xi}[-\psi(x-\bar{x})+\psi(0)] \\
& \quad \in-A(x) \zeta(\bar{y}, \hat{y}(x))+K+B \gamma(x, \bar{x})+H+K+H \subset H
\end{aligned}
$$

where $A(x)$ and $\zeta(\cdot)$ are as above, $B \in \partial_{x} L(\bar{x}, \bar{y} ; \tilde{\Lambda}, \tilde{M}, \tilde{\Pi}, \tilde{\Xi}), \gamma(\cdot)$ is a scale function from the invex hypothesis (b), $\psi:=\left(\psi^{(1)}, \ldots, \psi^{(s-l)}\right)$. Thus $\bar{x}$ is a strong minimum with respect to the cone $H$ of the outer problem of (WMM1) with additional constraints $\psi^{(j)}(x-\bar{x}) \leq 0(j=1,2, \ldots, s-l)$.

REMARK. To find an instance where the hypotheses of Theorems 1 and 2 hold, suppose that the vector function $\Theta(x)$ reaches a local weak minimum at $z=p$, 
subject to the constraint $-\gamma(x) \in \Gamma$, where the convex cone $\Gamma$ has interior points. Then there is no solution $x$ to

$$
\Theta(x)-\Theta(p) \in-\text { int } Q, \quad-\gamma(x) \in \text { int } \Gamma \text {. }
$$

If the vector function $(\Theta, \gamma)$ is convexlike (see [12]), then there exist multipliers $(\tau, \lambda) \in Q^{*} \times \Gamma^{*}$, not both zero, such that

$$
\tau^{T}[\Theta(x)-\Theta(p)]+\lambda^{T} \gamma(x) \geq 0
$$

for all $x$ near $p$. Note that convexlike (with differentiable) implies invex. If a Slater constraint qualification holds, that is $-\gamma(c) \in$ int $\Gamma$ for some $c$, then the multiplier $\tau \neq 0$. Then there is a matrix $Y$, with $Y$ in the matrix cone $S:=\{y: y(\Gamma) \subset Q\}$, such that $\Theta(x)+Y \gamma(x)$ reaches a weak minimum at $x=p$. It follows that $f(x, y):=$ $\Theta(x)+y \gamma(x)$ reaches a weak minimax at $(x, y)=(p, Y)$, with respect to the cones $Q$ and $S$.

Consider the hypotheses of Theorem 1, and assume Lipschitz functions, so that Clarke generalized subdifferentials are available. The stability condition of Proposition 1 holds, in particular, if the generalized subdifferentials $\xi$ of the active constraints are linearly independent, and then hypothesis (ii) of Theorem 1 follows by suitable construction of the adjoined constraints. The linear growth condition in Proposition 1 holds, omitting active constraints, if $-\gamma(p) \in$ int $S$. Hypotheses (iv) and (v) of Theorem 1 require Lagrange multipliers to be continuous in their parameters. If the problem is differentiable, it suffices to locally linearize it, obtaining a linear program, and then to assume that the dual linear program is stable to small perturbations; then the Lagrange multipliers, which are dual variables, have the required continuity. For a nonsmooth problem, the same construction applies to the linear programs obtained by replacing gradients of generalized subdifferentials. A similar remark applies to hypothesis (v). The remaining hypothesis (iii) is invexity; this holds if the vector of $-f(x, \cdot)$ and the active $h^{(i)}(\cdot)$ is invex, by suitable choice of quadratic terms in the adjoined $\phi^{(j)}$. Theorem 2 assumes (KKT) condition (a), and invexity conditions (b), to which the discussion of invexity for Theorem 1 also applies. Hypothesis (c) is made to ensure the pseudo-Lipschitzian property; see [13] for discussion of when this holds.

\section{Acknowledgement}

The authors thank two anonymous referees for their careful and detailed checking of the paper, and constructive comments. 


\section{References}

[1] A. M. Arthurs, Complementary variational principles, (Clarendon Press, Oxford, England, 1979).

[2] J. P. Aubin, 'Lipschitz behaviour of solutions to convex minimization problems', Math. Oper: Res. 9 (1984), 87-111.

[3] F. H. Clarke, Optimization and nonsmooth analysis, (Wiley, New York, 1983).

(4] B. D. Craven, 'Strong vector minimization and duality', Z. Angew. Math. Mech. 60 (1980), 1-5.

[5] B. D. Craven, 'Vector-valued optimization', in Generalized concavity in optimization and economics (eds. S. Schaible and W. T. Ziemba), (Academic Press, New York, 1981), 661-687.

[6] B. D. Craven, 'Nondifferentiable optimization by smooth approximation', Optimization 17 (1986), 3-17.

[7] B. D. Craven, 'A modified Wolfe dual for weak vector minimization', Numer: Funct. Anal. Optim. 10 (1989), 899-907.

[8] B. D. Craven, 'Quasimin and quasisaddlepoint for vector optimization', Numer: Funct. Anal. Optim. $11(1970), 45-54$.

[9] B. D. Craven, 'Convergence of discrete approximations for constrained minimization', J. Austral. Math. Soc. (Series B) 35 (1994), 50-59.

[10] B. D. Craven and D. V. Luu, "An approach to optimality conditions for nonsmooth minimax problems', University of Melbourne, Department of Mathematics, Preprint Series No. 8 (1993).

[11] B. D. Craven and D. V. Luu, 'Constrained minimax for a vector-valued function', Optimization 31 (1994), 199-208.

[12] V. Jeyakumar, 'Convexlike alternative theorem and mathematical programming', Optimization 16 (1985), 643-652.

[13] B. Mordukhovich, 'Stability theory for parametric generalized equations and variational inequalities via nonsmooth analysis', Trans. Amer: Math. Soc. 343 (1994), 609-657.

[14] S. M. Robinson, 'Stability theory for systems of inequalities, Part II: Differentiable nonlinear systems', SIAM J. Numer: Anal. 13 (1976), 497-513.

[15] R. T. Rockafellar, 'Lipschitzian properties of multifunctions', Nonlinear Anal. 9 (1985), 867-885.

[16] N. D. Yen, 'Stability of the solution set of perturbed nonsmooth inequality systems', Preprint. (International Centre for Theoretical Physics, Trieste, Italy, 1990).

Department of Mathematics and Statistics

University of Melbourne

Parkville, VIC 3052

Australia
Institute of Mathematics P.O. Box 631, Bo Ho

10000 Hanoi

Vietnam 